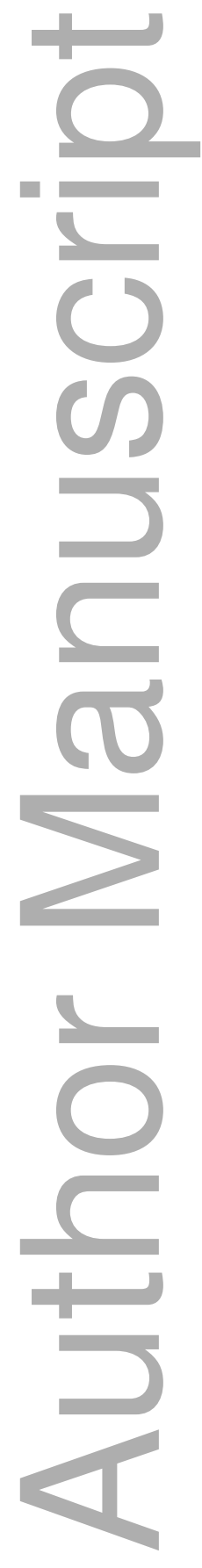

This is the author manuscript accepted for publication and has undergone full peer review but has not been through the copyediting, typesetting, pagination and proofreading process, which may lead to differences between this version and the Version of Record. Please cite this article as doi: $\underline{10.1111 / A N Z S .12149}$

This article is protected by copyright. All rights reserved 


\title{
NONPARAMETRIC ESTIMATION OF THE NUMBER OF DRUG USERS IN HONG KONG USING REPEATED MULTIPLE LISTS
}

\begin{abstract}
Richard M. HugGins ${ }^{1 *}$, PAul S.F.P. YiP ${ }^{2}$, JAKub StOKlos ${ }^{3}$
The University of Melbourne, The University of Hong Kong, The University of

$\square$

New South Wales

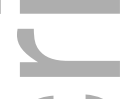

Summary

We update a previous approach to the estimation of the size of an open population when there are multiple lists at each time point. Our motivation is 35 years of longitudinal data on the detection of drug users by the Central Registry of Drug Abuse in Hong Kong. We develop a two-stage smoothing spline approach. This gives a flexible and easily implemented alternative to the previous method which was based on kernel smoothing. The new method retains the property of reducing the variability of the individual estimates at each time point. We evaluate the new method by means of a simulation study that includes an examination of the effects of variable selection. The new method is then applied to data collected by the Central Registry of Drug Abuse. The parameter estimates obtained are compared with the well known Jolly-Seber estimates based on single capture methods.
\end{abstract}

Key words: log-linear models; model selection; population size estimation; smoothing splines.

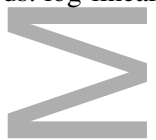

\section{Introduction}

One of the challenges facing a modern statistician is to modify classical methods so that they can be applied efficiently to big data sets. We update the approach of Liu et al. (2007) to the nonparametric estimation of the size of an open population from multiple lists using Poisson log-linear models. Our update enables us to apply the approach to a big data set consisting of 35 years of longitudinal data on detection of drug users accumulated by the Central Registry of Drug Abuse (CRDA) in Hong Kong. The CRDA within the Narcotics Division of Hong Kong monitors the numbers of drug abusers in Hong Kong through nine agencies, listed in Table 1. We consider annual data and record whether an individual was observed at least once in a given year by one of the nine agencies. The full data set contains

\footnotetext{
*Author to whom correspondence should be addressed.

${ }^{1}$ Department of Mathematics \& Statistics, The University of Melbourne, Victoria, 3010, Australia.

${ }^{2}$ Department of Social Work and Social Administration, Faculty of Social Sciences, The University of Hong Kong, Hong Kong, Hong Kong SAR.

${ }^{3}$ School of Mathematics and Statistics and Evolution \& Ecology Research Centre, The University of New South Wales, New South Wales 2052, Australia.

Acknowledgment. The authors would like to thank the Central Registry of Drug Abuse within the Narcotics Division of Hong Kong for motivating the problem considered here.
} 
observations on the detection of approximately 150,000 individuals from 1977 to 2011 . These data have previously been examined by CRDA (Policy 21 Limited 2013a, b) who considered log-linear models using several lists for each year. However they do not consider the entire nine lists that we consider here and the estimates they used were not smoothed. The fact that log-linear models are being used in practice to analyse repeated multiple lists, and are part of a decision making process, has motivated us to update the previous approach to the analysis of such data as developed by Liu et al. (2007). In this approach a kernel smoothing method was applied to a subset of the data considered here. In this analysis the histories of the drug users was not available; here we consider full individual histories.

TABLE 1

The nine agencies reporting to the Central Registry of Drug Abuse in Hong Kong.

CS Drug Addiction Treatment Centre Programme of Correctional Services Department PF Hong Kong Police Force

MTP Methadone Treatment Programme under Department of Health

SWD Social Welfare Department

HA Substance Abuse Clinics under Hospital Authority / Hospitals / Clinics

NGO Non-government Organizations (NGOs) - Drug Treatment and Rehabilitation Centres

NGO2 NGOs - Out-Reaching Teams / Integrated Services Centres

NGO3 NGOs - Counselling Centres for Psychotropic Substance Abusers

NGO4 NGOs - Centres for Drug Counselling

The use of Poisson log-linear models to estimate the size of a closed population using capture data has a long history (Fienberg 1972; Cormack 1989) and these models are particularly appropriate for the analysis of data arising from multiple lists where there is no natural ordering of the lists. There is an extensive literature on the use of capturerecapture methods in estimating disease prevalence (International Working Group for Disease Monitoring and Forecasting 1995a, b) and log-linear models have become a standard method of estimating the prevalence of drug use. See for example European Monitoring Centre for Drugs and Drug Addiction (1997, Part III) and Hickman et al. (2002) and the references therein. Our approach involves a philosophy similar to the robust design methods of Pollock (1982) that involve primary and secondary occasions. In our application, the primary occasions are the years and the detections of drug users within each year can be regarded as the secondary occasions. In the first stage, the log-linear model is applied to all sets of secondary occasions so as to estimate the population size. In the second stage the resulting estimates are pooled to produce smooth estimates with reduced variability.

We assume individuals behave independently of one another and that for each observation method or list all individuals in the population have the same probability of being observed by that method. In practice this assumption may be relaxed by stratification of the 
population and it is implicit that all individuals can be observed by all methods. However the probabilities of being observed by the different methods can vary. Clearly, there may be an interaction or dependence between membership in different lists in each observation period and the log-linear model accommodates this. Dependence between observation periods can also occur although we do not model this explicitly here: even with only four lists there are 16 possible states at each primary occasion so the transition matrix is at least $16 \times 16$ in that case and is $17 \times 17$ if one includes an absorbing state for the removal of drug users from the observable population. With nine lists there are 512 states and the resulting transition matrix is enormous. The availability of longitudinal data does, however, allow the estimation of the numbers of drug users on the basis of each observation method and the aggregate data using the well known Jolly-Seber method (Jolly 1965; Seber 1965).

Whilst log-linear models are well known, for convenience we briefly review their application to cross-sectional data in Section 2. These cross sectional estimators are used in the first stage of our estimator to produce marginal estimates of the population size at each observation time. Previously Liu et al. (2007) presented a two stage approach that used kernel smoothing methods in a second stage to produce smooth estimators of the population size. In our two stage approach described in Section 3 we smooth the marginal estimates produced in the first stage by means of a smoothing spline approach that can be implemented using the gam function from the mgcv package (Wood 2006) in R (R Core Team 2016), although in the simulations and application discussed below we used our own programs. In Section 3 we also derive approximate standard errors of the smooth estimators. Our simulations revealed a slight bias in the estimated standard errors of the smooth estimators and we propose a bootstrap procedure to alleviate this problem in Section 3.2.

Part of the purpose of this work is to assess to what extent the log-linear model may be applied to data such as that arising from the CRDA. We could collapse nine observation methods into four as was done in Liu et al. (2007), however, this could mask differences between the various NGO's. In this paper we consider the full nine methods so as to demonstrate the method can indeed be used in this case. The issue of model selection then arises. Liu et al. (2007) conducted a preliminary analysis and for their four list model considered all three-way interactions. This is also possible in our setting but the large numbers of parameters estimated to fit these models can inflate the variance of the resulting estimators. This suggests that some variable selection should be done. For simplicity here we consider stepwise procedures based on the AIC that are easily implemented using stepAIC from the MASS package (Venables and Ripley 2013) in R. We examine the effects of variable selection for several possible types of list dependence using simulations in Section 4.1 and assess the performance of the bootstrap estimates of the standard errors in Section 4.2. We 
apply our method to the CRDA data in Section 5 and compare the estimates with the biascorrected Jolly-Seber estimates using only arrests by the police as captures and with all the capture types pooled. We finish with some discussion relating our method to composite likelihood in Section 6.

\section{The Cross-Sectional Log-linear Estimators}

In order to make this paper self contained, we briefly review the log-linear approach in our context. In each year each individual in the population of interest may appear on any of $S$ lists. A cell is then an $S$ vector of 0's and 1's that represent the lists upon which the individual appeared. Let $c$ denote a cell, $\boldsymbol{0}$ the null cell consisting of $S$ zeroes, $\mathcal{C}$ the set of all cells, $\mathcal{C} \backslash \mathbf{0}$ the set of all cells with the null cell omitted. We will refer to an individual as being "in" a cell $c$ if $c$ represents the set of lists on which the individual appeared. The observed data is the number of individuals in each cell apart from the null cell count which is not observed. Let $\mu_{c, t}$ denote the expected number of individuals in cell $c$ at time $t$. In the $\log$-linear model, $\mu_{c, t}$ is of the form $\log \mu_{c, t}=X_{c t}^{\top} \boldsymbol{\theta}_{t}$ where $X_{c t}$ is a vector of predictors constructed from the lists that can include the main effects of each list as well as interactions between lists, which may vary with $t$, and $\boldsymbol{\theta}_{t}$ is a vector of parameters. Crucially, the model is constructed so that the intercept term is the mean number of unobserved individuals. That is, $\log \mu_{\mathbf{0} t}=\theta_{0 t}$ where $\theta_{0}$ is the first entry of $\boldsymbol{\theta}_{t}$. By ignoring some usually high order interactions the model parameters are identifiable.

To illustrate this, consider the simple two list problem. In this setting the cells are $(0,0),(1,0),(0,1),(1,1)$ and the full model is

$$
\left(\begin{array}{l}
\log \mu_{00, t} \\
\log \mu_{10, t} \\
\log \mu_{01, t} \\
\log \mu_{11, t}
\end{array}\right)=\left(\begin{array}{cccc}
1 & 0 & 0 & 0 \\
1 & 1 & 0 & 0 \\
1 & 0 & 1 & 0 \\
1 & 1 & 1 & 1
\end{array}\right)\left(\begin{array}{c}
\theta_{0 t} \\
\theta_{1 t} \\
\theta_{2 t} \\
\theta_{12 t}
\end{array}\right)
$$

which involves four parameters for each $t$. In order for $\theta_{0 t}$ to be estimable, the interaction term must be omitted and the resulting main effects model fitted to the observed data. With more than two types of observation the model may be extended and more interaction terms included, but for $\theta_{0 t}$ to be identifiable some, typically high order, interactions need to be omitted. In practice, preliminary modelling is conducted to determine these. Note that $\theta_{0 t}$ is an intercept that appears in each $\mu_{c t}$ so that the design matrix contains a column of 1's.

The log-linear model has the advantage that it is a generalised linear model which may be fitted to data using software that is readily available in many statistical computing packages. Let $\widehat{\boldsymbol{\theta}}_{t}$ be the resulting estimator of $\boldsymbol{\theta}_{t}$ and let $\boldsymbol{\Sigma}_{\boldsymbol{\theta}}(t)$ be its covariance matrix 
whose estimator is denoted by $\widehat{\boldsymbol{\Sigma}}_{\boldsymbol{\theta}}(t)$. Following Liu et al. (2007) the population size at time $t_{j}, N\left(t_{j}\right)$, is estimated by $\widehat{N}\left(t_{j}\right)=\exp \left(\widehat{\theta}_{0 t_{j}}\right)+n\left(t_{j}\right)$, where $n\left(t_{j}\right)$ is the total number of individuals appearing on any of the lists at time $t_{j}$. Computing the standard errors for this estimator is difficult and Liu et al. (2007) only gave an upper bound. However, it is well known that when the design matrix contains a column of 1's, fitting the model to the data with the null cell omitted yields $n(t)=\sum_{\mathcal{C} \backslash 0} \widehat{\mu}_{c t}$ (also see the remark following (2) below). Now, $\mathrm{E}\{N(t)\}=\sum_{\mathcal{C}} \mu_{c t}$ where the sum is now over all cells including the empty cell. Consequently $\widehat{N}(t)=\sum_{\mathcal{C}} \widehat{\mu}_{c t}=\sum_{\mathcal{C}} \exp \left(X_{c t}^{\top} \widehat{\boldsymbol{\theta}}_{t}\right)$. Let $\boldsymbol{X}_{t}$ be the design matrix that we assume contains a column of 1's. As noted above, this design matrix may depend on $t$ as there may be different interactions between the observation methods at different times. The predicted values are $\exp \left(\boldsymbol{X}_{t} \widehat{\boldsymbol{\theta}}_{t}\right)$. The usual Taylor series approximation gives $\exp \left(\boldsymbol{X}_{t} \widehat{\boldsymbol{\theta}}_{t}\right) \approx \exp \left(\boldsymbol{X}_{t} \boldsymbol{\theta}_{t}\right)+\exp \left(\boldsymbol{X}_{t} \boldsymbol{\theta}_{t}\right) \boldsymbol{X}\left(\widehat{\boldsymbol{\theta}}_{t}-\boldsymbol{\theta}_{t}\right)$ and hence as usual, $\operatorname{cov}\left\{\exp \left(\boldsymbol{X}_{t} \widehat{\boldsymbol{\theta}}_{t}\right)\right\} \approx \exp \left(\boldsymbol{X}_{t} \boldsymbol{\theta}_{t}\right) \boldsymbol{X}_{t} \boldsymbol{\Sigma}_{\boldsymbol{\theta}}(t) \boldsymbol{X}_{t}^{\top} \exp \left(\boldsymbol{X}_{t} \boldsymbol{\theta}_{t}\right)^{\top}$ so that

$$
\begin{aligned}
\sigma_{t}^{2} & =\operatorname{var}\{\widehat{N}(t)\}=\mathbf{1}^{\top} \operatorname{cov}\left\{\exp \left(\boldsymbol{X}_{t} \widehat{\boldsymbol{\theta}}_{t}\right)\right\} \mathbf{1} \\
& \approx \mathbf{1}^{\top} \exp \left(\boldsymbol{X}_{t} \boldsymbol{\theta}_{t}\right) \boldsymbol{X}_{t} \boldsymbol{\Sigma}_{\boldsymbol{\theta}}(t) \boldsymbol{X}_{t}^{\top} \exp \left(\boldsymbol{X}_{t} \boldsymbol{\theta}_{t}\right)^{\top} \mathbf{1}
\end{aligned}
$$

All these quantities may be easily computed after fitting a generalised linear model.

Remark Note that $\boldsymbol{\theta}_{t}$ is the solution of

$$
S_{t}\left(\boldsymbol{\theta}_{t}\right)=\boldsymbol{X}_{t}^{\top}\left(\boldsymbol{y}_{t}-\boldsymbol{\mu}_{t}\right)=0
$$

where $\boldsymbol{y}_{t}=\left\{y_{c, t}, c \in \mathcal{C} \backslash 0\right\}$ and $\boldsymbol{\mu}_{t}=\left\{\mu_{c, t}, c \in \mathcal{C} \backslash 0\right\}$ and as the first column of $\boldsymbol{X}_{t}$ is a column of 1's, this gives $n(t)=\sum_{\mathcal{C} \backslash 0} y_{c, t}=\sum_{\mathcal{C} \backslash 0} \mu_{c, t}$, which was used above. This also holds for the quasi-Poisson approach, but not the negative binomial. Thus for the negative binomial it is necessary to work directly with $\exp \left(\widehat{\theta}_{0 t_{j}}\right)+n\left(t_{j}\right)$.

Remark It is clear from the above discussion that the log-linear method estimates $\mathrm{E}\{N(t)\}$. On the other hand, the Jolly-Seber method estimates $N(t)$, which Jolly (1965) refers to as a conditional parameter. Consequently when we compare the two estimators below one should keep this distinction in mind.

\section{Smoothing the Log-Linear Estimators}

Smoothing spline estimators are useful in the analysis of noisy data as they give a smooth representation of the mean function and by what is in effect a pooling of observations can dramatically reduce the variability of the estimates. Smoothing spline estimators have an 
advantage over those obtained from kernel smoothing in that they are easier and faster to compute.

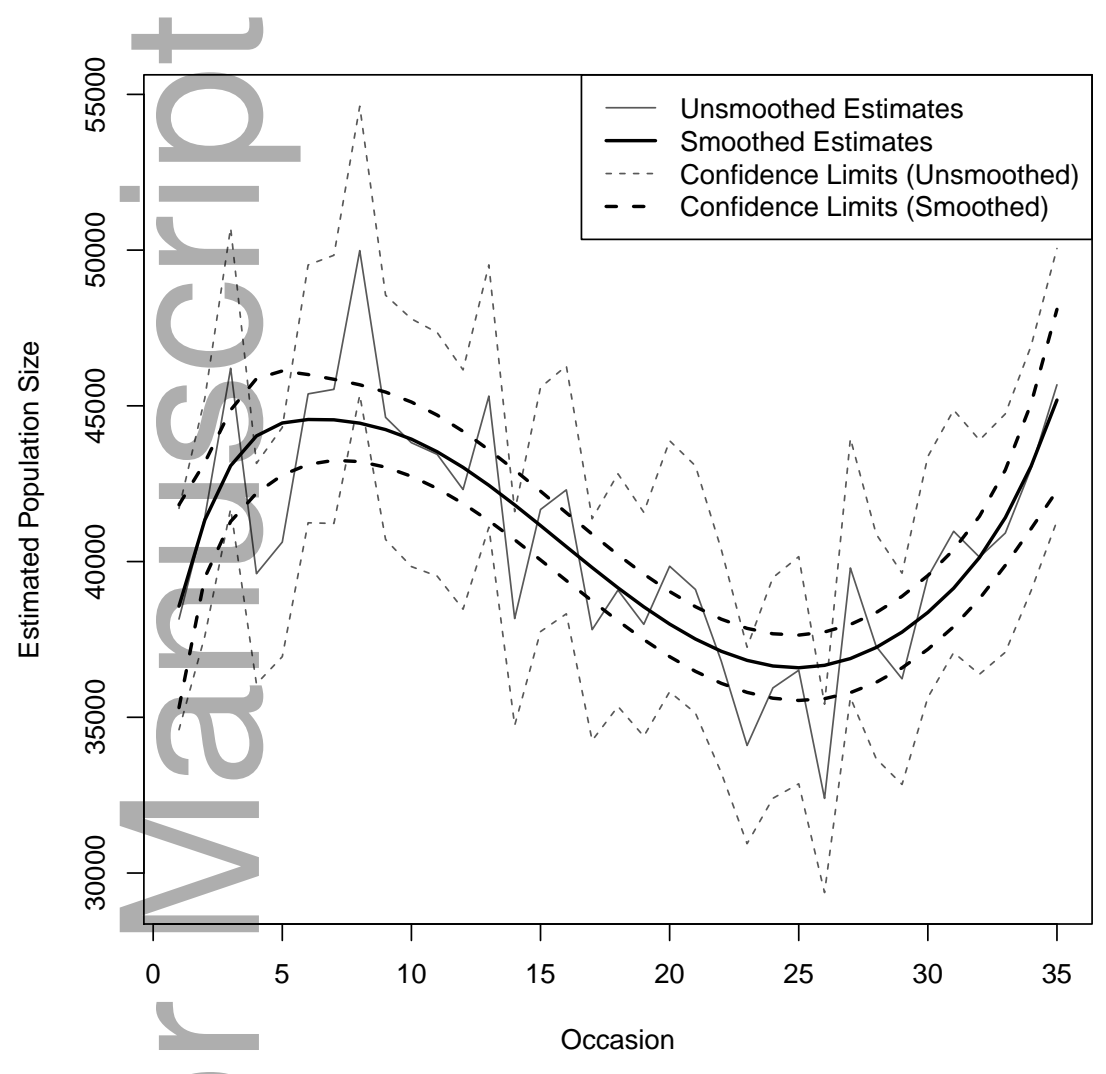

Figure 1. Log-linear estimates (solid lines) of the mean population size $\mathrm{E}\left(N_{t}\right)$ and the associated confidence intervals (dashed lines) from the raw unsmoothed estimates and the smoothed estimates for simulated data over 35 occasions.

\subsection{Penalised Splines}

Let $t_{1}, \ldots, t_{J}$ be the times at which observations were made. The raw estimates $\widehat{N}\left(t_{j}\right)$ are based on marginal data collected at each $t_{j}$ and do not incorporate the individual capture histories. In our approach we suppose that $\mathrm{E}\{N(t)\}=\boldsymbol{w}(t)^{\top} \boldsymbol{\beta}$ for some basis functions $\boldsymbol{w}(t)$, and we estimate $\boldsymbol{\beta}$ using weighted least squares. Let $\boldsymbol{W}$ be a matrix with $j$ th row $\boldsymbol{w}\left(t_{j}\right)^{\top}$. Then

$$
\widetilde{\boldsymbol{\beta}}_{\lambda}=\left(\boldsymbol{W}^{\top} \boldsymbol{W}+\lambda \boldsymbol{P}\right)^{-1} \boldsymbol{W}^{\top} \widehat{N}\left(t_{j}\right)
$$


for a penalty matrix $\boldsymbol{P}$ and smoothing parameter $\lambda$. Thus our smooth estimator is $\tilde{N}_{\lambda}(t)=$ $\boldsymbol{w}(t)^{\top} \widetilde{\boldsymbol{\beta}}_{\lambda}$. To compute this, we generate the basis $\boldsymbol{W}$ as a B-spline basis, take $\boldsymbol{P}$ to be a P-spline penalty matrix (Eilers \& Marx 1996) and use generalised cross validation (GCV, see Section 3.2.3 of Wood 2006) to estimate $\lambda$. For known $\lambda$, the variance of the estimator is

$$
\operatorname{var}\left(\widetilde{\boldsymbol{\beta}}_{\lambda}\right)=\left(\boldsymbol{W}^{\top} \boldsymbol{W}+\lambda \boldsymbol{P}\right)^{-1} \boldsymbol{W}^{\top} \boldsymbol{\Sigma}(\widehat{\boldsymbol{N}}) \boldsymbol{W}\left(\boldsymbol{W}^{\top} \boldsymbol{W}+\lambda \boldsymbol{P}\right)^{-1},
$$

where $\boldsymbol{\Sigma}(\widehat{\boldsymbol{N}})$ is the covariance matrix of the raw estimators. Write $\widetilde{\boldsymbol{N}}_{\lambda}=$ $\left(\tilde{N}_{\lambda}\left(t_{1}\right), \ldots, \widetilde{N}_{\lambda}\left(t_{J}\right)\right)^{\top}$. Then, again for known $\lambda, \operatorname{var}\left(\widetilde{\boldsymbol{N}}_{\lambda}\right)=\boldsymbol{W} \operatorname{var}\left(\widehat{\boldsymbol{\beta}}_{\lambda}\right) \boldsymbol{W}^{\top}$. To employ these formulae we need to estimate $\boldsymbol{\Sigma}(\widehat{\boldsymbol{N}})$ and account for the variability in the estimate of $\lambda$, which is obtained by means of GCV. Write $\widetilde{\boldsymbol{\beta}}$ and $\widetilde{N}_{t}$ for the resulting penalised spline estimators. Even if $\boldsymbol{\Sigma}(\widehat{\boldsymbol{N}})$ were known, the variance given by (3) evaluated at the estimated value of $\lambda$ still underestimates the variance of $\widetilde{\boldsymbol{\beta}}$ as it does not take the variability of $\lambda$ into account. We address this problem in Section 3.2. To illustrate the effectiveness of smoothing, in Figure 1 we plot both the raw estimates and the smooth estimates for some simulated data along with the associated $95 \%$ confidence intervals, computed using the bootstrap (see Section 3.2) for the smooth estimates. It is clear from this figure that smoothing can substantially reduce the variability of the estimators. See also the simulation results of Section 4 where the "Nominal" method has standard errors computed according to (3) assuming independent $\widehat{N}_{t}$.

Remark To estimate $\boldsymbol{\Sigma}(\widehat{N})$ directly, we can make use of estimators of $\operatorname{var}\left\{\widehat{N}\left(t_{j}\right)\right\}$ from (1). We also need to estimate $\operatorname{cov}\left(\widehat{N}_{t_{j}}, \widehat{N}_{t_{k}}\right)$. Our initial approach was to assume the independence of the estimators but simulations revealed that this underestimated the true variability. Fitting an autoregressive model of the form $\mathrm{E}\{\widehat{N}(t) \mid \widehat{N}(t-1)\}=\mu_{t}+\alpha \widehat{N}(t-$ 1) to the estimated population sizes and approximating $\boldsymbol{\Sigma}(\widehat{\boldsymbol{N}})$ by the resulting covariance matrix did not improve the estimated standard errors. We were unable to find an analytic method of estimating the standard errors that performed well in simulations.

\subsection{Bootstrap Estimates of Standard Errors}

Wood (2006) employed a Bayesian approach (Marra \& Wood 2012) to estimate the standard errors of their smoothing spline estimators. However, we prefer to use a bootstrap. Liu et al. (2007) observed in their simulations for the kernel estimator that the estimated variances assuming independence were slightly too large if there were dependencies. They interpreted this phenomenon as being due to some individuals being common to the populations present at both times and they proposed the use of a bootstrap, which we also employ. The bootstrap can be quite slow when kernel estimators are employed, but for our 
procedure it is far more practical since penalised spline estimators can be computed very quickly. Because we still need to estimate the covariance matrix $\boldsymbol{\Sigma}(\widehat{N})$, which appears to be analytically intractable, the bootstrap appears the best solution. We use a bootstrap procedure that consists of simulating from a multinomial distribution on each occasion using the empirical probabilities of each combination of lists on each occasion and the numbers of drug users observed on that occasion. Suppose there are $n_{t}$ individuals observed on occasion $t$ and $n_{t c}$ of these are observed in cell $c$. Then we set $p_{t c}=n_{t c} / n_{t}$ and our bootstrap sample is then multinomial $\left(n_{t}, p_{t c}\right), c \in \mathcal{C} \backslash 0$. This bootstrap ignores behavioural response, but performed adequately in our simulations. When computing the estimates from the bootstrap samples we recomputed the smoothing parameter $\lambda$ for each sample.

To employ the bootstrap we can compute the standard deviations (denoted by bse $e_{t}$ ) of the estimated $\widehat{N}_{t}$ over the bootstrap samples and substitute these for the standard errors in the usual large sample formula for a confidence interval: $\widetilde{N}_{t} \pm z_{\alpha / 2} b s e_{t}$. Alternatively we can use the bootstrap-t approach based on $T=\left(\widetilde{N}_{t}-N_{t}\right) / s e_{t}$ where $s e_{t}$ is the estimated standard error of $\widetilde{N}_{t}$ assuming independence. Then if $t_{\alpha}^{*}$ is the $100 \alpha$ th percentile of the empirical distribution of $T^{*}=\left(\widetilde{N}_{t}^{*}-\widetilde{N}_{t}\right) / s e_{t}^{*}$, where ${ }^{*}$ denotes the estimate from the bootstrap sample, the bootstrap- $t$ confidence interval is given by $\left(\widetilde{N}_{t}-t_{1-\alpha / 2}^{*} s e_{t}, \widetilde{N}_{t}-t_{\alpha / 2}^{*} s e_{t}\right)$, see Section 2.4 of Davison \& Hinkley (1997).

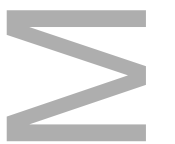

\section{Simulations}

We ran a simulation study to assess the performance of the estimators, including the model selection step and the coverage achieved by the bootstrap confidence intervals. In the simulations we supposed the population to be a first order Poisson autoregressive process $N_{t} \sim$ Poisson $\left(\mu_{t}+\alpha N_{t-1}\right), t=1, \ldots, J$ with $0<\alpha<1$, and initial size $N_{0}$. To examine model selection in Section 4.1 we took $\mu_{t}=N_{0}(1-\alpha)$ for each $t$ and to examine the performance of the bootstrap estimates of the standard errors we took $\mu_{t}=$ $N_{0}(1-\alpha)\{1+\sin (t / 5) / 10\}$. That is, $\alpha$ is the probability that a drug user is still a drug user in the next year and in year $t$ there were a mean number $\mu_{t}$ of new drug users entering the population. For simplicity we supposed there were four lists and rather than model the transitions between states we took the number of individuals in each state to have a multinomial $\left(N_{t}, \boldsymbol{p}\right)$ distribution where again for simplicity the vector of probabilities $\boldsymbol{p}$ did not depend on $t$. To be consistent with our real-data example, we took $N_{0}=40,000$ and $J=35$. To compute the multinomial probabilities we considered a model with twoway interactions, computed the appropriate design matrix $\boldsymbol{X}$ and computed the means $\boldsymbol{\mu}=$ $\exp (\boldsymbol{X} \boldsymbol{\beta})$. We then took the probabilities to be $\boldsymbol{p}=\boldsymbol{\mu} / \sum \boldsymbol{\mu}$. Note that in the log-linear model the first term in $\boldsymbol{\beta}$ is common to all entries of $\boldsymbol{\mu}$ and hence cancels out in computing $\boldsymbol{p}$. 


\subsection{Model Selection}

\section{TABLE 2}

\begin{tabular}{cc}
\multicolumn{2}{c}{\begin{tabular}{c} 
Values of $\boldsymbol{\beta}$ used in the simulation study of model selection methods. \\
\hline \hline Parameter
\end{tabular}} \\
\hline $\boldsymbol{\beta}_{0}$ & $10.0,-1.8,-1.8,-1.8,-1.8,0,0,0,0,0,0$ \\
$\boldsymbol{\beta}_{P}$ & $10.0,-1.8,-1.8,-1.8,-1.8,0.5,0.5,0.5,0.5,0.5,0.5$ \\
$\boldsymbol{\beta}_{N}$ & $10.0,-1.8,-1.8,-1.8,-1.8,-0.5,-0.5,-0.5,-0.5,-0.5,-0.5$ \\
$\boldsymbol{\beta}_{B}$ & $10.0,-1.8,-1.8,-1.8,-1.8,0.5,0.5,0.5,-0.5,-0.5,-0.5$ \\
$\boldsymbol{\beta}_{M}$ & $10.0,-1.8,-1.8,-1.8,-1.8,0.5,0.5,0.5,0,0,0$ \\
\hline
\end{tabular}

As there are many interactions to be considered we conducted simulations to investigate some possible stepwise model selection methods. We considered five values for $\boldsymbol{\beta}$ that we list in Table 2. These correspond to no interactions between the lists, a positive interaction, a negative interaction, a mixture of positive and negative interactions and a mixture of positive and zero interactions. First we investigated the effects of model selection on the raw estimators. We considered two approaches based on the AIC: A stepwise procedure starting with the full two-way interaction model (essentially backward selection) and a similar approach that started with the intercept only model and considered the main effects and two factor interactions (essentially forward selection). Both approaches were implemented using stepAIC from the R-package MASS (Venables and Ripley 2013). A third approach used was to fit the main effects and two factor interactions with no model selection. The results of 1,000 simulations for each value of $\boldsymbol{\beta}$ are given in Table 3 for the raw estimators. Both the bias and root mean square error were computed in terms of distances from the common mean $N_{0}=40,000$. The results show that stepwise selection from the model with main effects and two-way interactions performed well in all scenarios, and although not the best in all scenarios it never had the very poor performance exhibited by the other two procedures in some cases. The approach without selection performed poorly when the true model only involved main effects or when some interactions were zero. However it was much faster to compute. The forward selection approach had a huge bias when some of the interactions were negative. These, admittedly limited, results strongly suggest that careful model selection is required, but backward stepwise selection starting from the full main effects and two-way interaction model appears to perform well. Of course there could be three-way interactions but with a large number of lists, modelling these becomes computationally intractable. 
TABLE 3

The average bias (Bias) and averages of the root mean square errors (RMSE) for the raw estimators over the four simulations scenarios for three methods of model determination.

\begin{tabular}{lrrrrrr}
\hline \hline Model & \multicolumn{5}{c}{ Model selection method } \\
\hline & $\begin{array}{c}\text { Stepwise from } \\
\text { Full Model }\end{array}$ & $\begin{array}{c}\text { Stepwise from } \\
\text { Intercept Model }\end{array}$ & No selection \\
& Bias & RMSE & Bias & RMSE & Bias & RMSE \\
\hline & 77.3 & 894.4 & 77.4 & 695.2 & 106.6 & 1491.2 \\
\hline No interactions $\left(\boldsymbol{\beta}_{0}\right)$ & 9.1 & 850.9 & 9.1 & 850.9 & 9.1 & 850.9 \\
Positive interactions $\left(\boldsymbol{\beta}_{P}\right)$ & -79.9 & 2826.0 & 10521.1 & 13184.4 & -97.9 & 2754.9 \\
Negative interactions $\left(\boldsymbol{\beta}_{N}\right)$ & 72.5 & 1408.8 & 72.3 & 1409.4 & 72.5 & 1408.8 \\
$\begin{array}{l}\text { Positive and } \\
\text { negative interactions }\left(\boldsymbol{\beta}_{B}\right)\end{array}$ & 0.0 & 877.6 & -1.50 & 837.3 & 5.30 & 1105.1 \\
$\begin{array}{l}\text { Positive and } \\
\text { zero interactions }\left(\boldsymbol{\beta}_{M}\right)\end{array}$ & & & & & & \\
\hline
\end{tabular}

\section{TABLE 4}

Average coverage probabilities (cover) and average widths (ave.w) of the confidence intervals for 100 simulation with 100 bootstrap replicates per simulation.

\begin{tabular}{lcccccccc}
\hline \hline & & \multicolumn{9}{c}{$\alpha$} \\
\hline & & 0.2 & \multicolumn{2}{c}{0.4} & \multicolumn{2}{c}{0.6} & \multicolumn{2}{c}{0.8} \\
\hline \multicolumn{1}{c}{ cover } & ave. w & cover & ave.w & cover & ave.w & cover & ave.w \\
\hline Raw & 0.95 & 7601 & 0.95 & 7612 & 0.95 & 7610 & 0.95 & 7657 \\
Nominal & 0.91 & 2997 & 0.90 & 2949 & 0.89 & 2888 & 0.89 & 2832 \\
Bootstrap & 0.93 & 3214 & 0.92 & 3171 & 0.93 & 3141 & 0.93 & 3145 \\
Bootstrap- $t$ & 0.94 & 3225 & 0.93 & 3151 & 0.94 & 3101 & 0.93 & 3093 \\
\hline
\end{tabular}

\subsection{Bootstrap Standard Errors}

To assess the bootstrap estimators we considered a model that had all the main effects some negative two-way interactions and some zero interactions. Explicitly we took $\boldsymbol{\beta}=$ $(10.0,-1.8,-1.8,-1.8,-1.8,-0.5,-0.5,-0.5,0.0,0.0,0.0)$. We then computed the loglinear estimators for the model that included all two-way interactions. In Table 4 we report the average coverage probabilities and average widths of confidence intervals over 100 simulations using 100 bootstrap samples in the setting of Section 4.1. That is, 3,500 individual intervals were considered for the purpose of estimating the coverage probabilities. These probabilities indicate that the coverage of both types of bootstrap intervals is close to but slightly lower than the nominal 95\%. The Nominal intervals, which use the uncorrected formulae based on (3), have a poorer coverage. Moreover, as foreshadowed by Figure 1 the smooth estimators gave much narrower confidence intervals than the raw estimators. 


\section{Application}

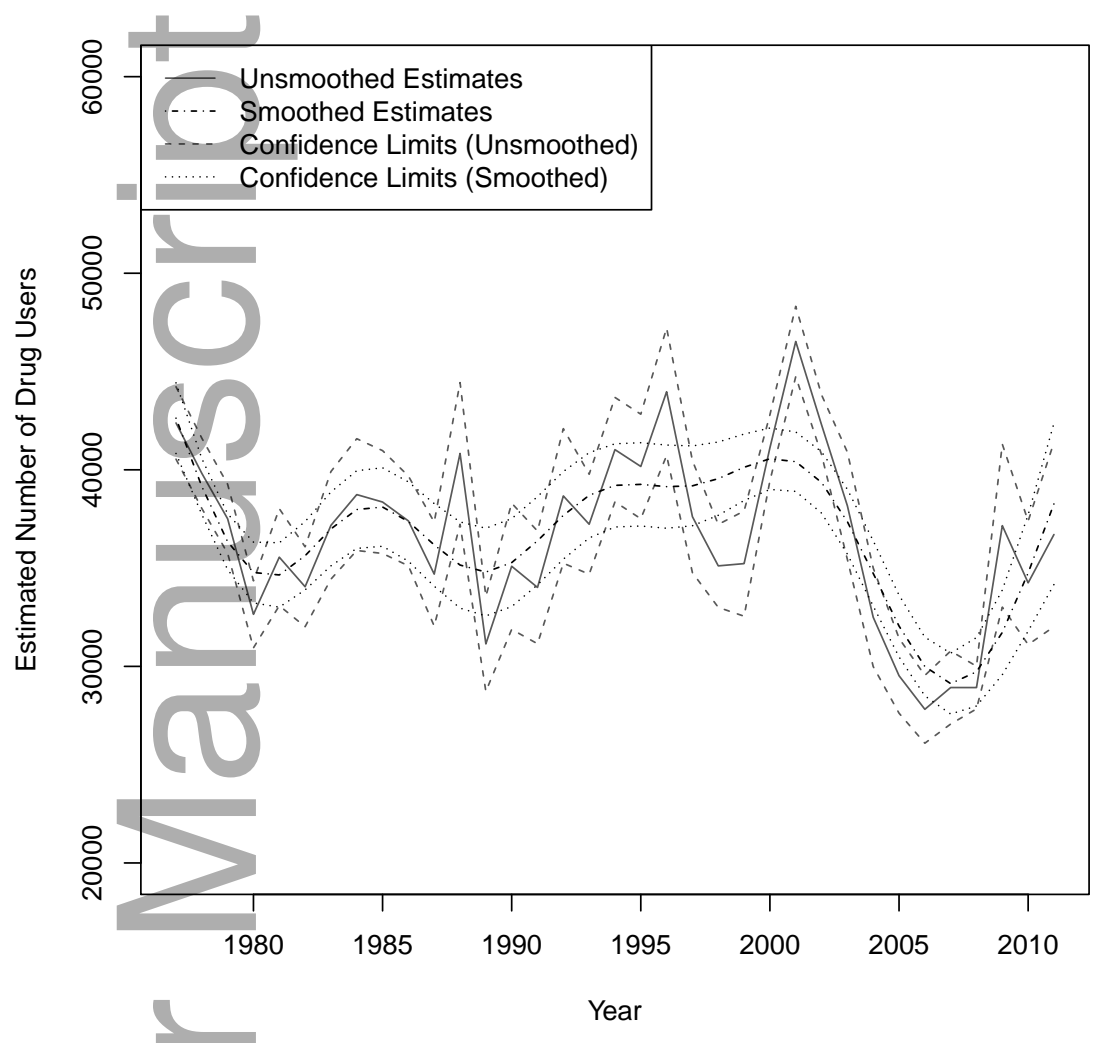

Figure 2. The unsmoothed log-linear estimates (solid line) and associated smooth estimates (dot-dashed line) of the annual numbers of drug users in Hong Kong with associated bootstrap confidence bounds (here the dashed lines correspond to confidence bounds for the unsmoothed estimates and dotted lines correspond confidence bounds for the smoothed estimates).

To illustrate our approach, we fitted the model to annual observations where an individual is taken to be on a list if they appear on that list at least once during the year. In accordance with our simulation results we used backward stepwise model selection starting from a model including all main effects and two-way interactions and conducted model fitting for each year. We used 9 knots to compute the spline basis and estimated the smoothing parameter using GCV. The bootstrap was used to estimate the standard errors. We computed estimates, which were similar for these data, using all methods. In Figure 2 we plot the unsmoothed and smoothed estimates of the numbers of drug users in Hong Kong. The smooth estimates make interpretation easier: The number of drug users was consistently 
around 35,000 for the first 25 or so years of the period under study then declined slightly for the last 10 years. In Figure 3 we plot the unsmoothed estimators along with the bias-corrected Jolly-Seber estimators computed using the aggregate data and using only the police data separated out from the aggregate data. These are consistent towards the centre of the data apart from a period around 2000-2002 where there is a marked peak in the Jolly-Seber estimator using the police data alone. They also differ in 2010 when both the Jolly-Seber estimates were less than the log-linear estimate and towards the start of the data when again the JollySeber estimates are smaller than those arising from the log-linear model. There was a peak in the raw estimates around 2001-2002. It is not clear what caused this peak, however, due to its form, the Jolly-Seber estimator can produce peaks if most of the individuals captured on a given occasion had not been previously captured. This could arise through a change in policing strategies for example. The fact that the Jolly-Seber estimate based on police data differs from the log-linear estimate in 2001-2002 suggests more sophisticated modelling is required. The difference in 2010 is also of interest as anecdotal evidence is that the number of drug users is falling, which agrees with the two Jolly-Seber estimates. However, the log-linear estimates increase in 2010, and although the estimated confidence intervals are wide they do not overlap with those arising from the Jolly-Seber estimates. Again a more sophisticated analysis is required to resolve this conundrum. We expand on this comment in the discussion.

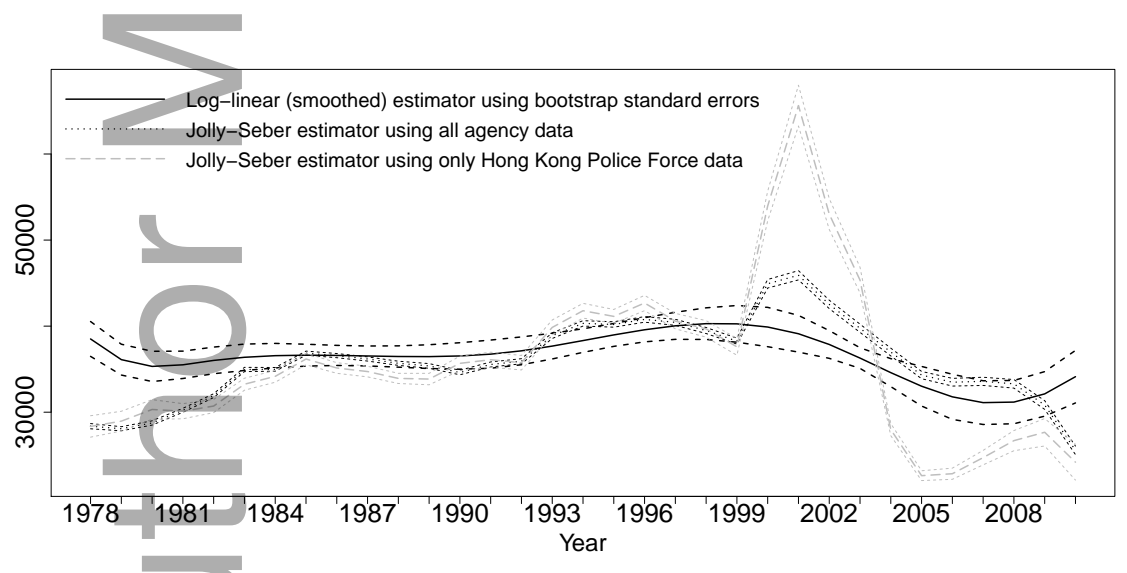

Figure 3. The smooth estimates and the Jolly-Seber estimates of the numbers of drug users based on the pooled data and on the police data alone.

\section{Discussion}

We have improved the method of Liu et al. (2007) so that it is now more practical and more easily applied to large data sets. The approach applies a closed population estimator 
to multiple lists at each time point. In our present application these lists are formed from data aggregated over a year. It is obviously possible that individuals may enter and leave the population during the year so this requirement is unlikely to be strictly met. The method could be modified by using smaller intervals, such as months or weeks, wherein the population of drug users is closer to being closed. Potentially, a local estimation approach along the lines of Huggins \& Yip (1999) could be developed. The use of the bootstrap to estimate standard errors means that the approach can be applied to marginal data. The current approach could be easily improved bystratifying the drug users into homogeneous groups.

Underestimation of the standard error using the analytic formula is due to not taking into account the variability in the smoothing parameter and the dependence between captures on different occasions. The first can be overcome using the approach of (Marra \& Wood 2012) but the second is more difficult as it involves the estimation of the Poisson autoregressive parameter from the estimated population sizes which does not seem feasible. This may perhaps be overcome by working more directly with the observed histories using pairwise composite likelihood for example. Because this would detract from the simplicity of the present estimator we defer a consideration of this issue and instead use the bootstrap. When our approach is used computation of the estimates is generally relatively fast whence the bootstrap is feasible. However if one conducts variable selection at each stage the computations become more demanding and the bootstrap. Liu et al. (2007) used an upper bound on the standard errors of the raw estimates, which gave reasonable estimates of the standard errors of the smooth population size estimators.

In our application we noted a difference between the log-linear and Jolly-Seber estimates. One possible explanation for the difference is that the assumptions required for one or both approaches have been be violated. In particular, the log-linear approach requires that all methods access the same population. We remark that the use of EM-algorithm methods to circumlocute this requirement is possible in the setting in which the populations do not overlap at all (see Zwane et al. (2004)).

The discrepancies between the methods, as disclosed by our analysis, are somewhat worrisome. On the other hand the fact that there are discrepancies could provide illuminating insights about the data and our analysis thus has the practical advantage of revealing this possibility. The interaction terms in the log-linear model may give some guidance on the reconciliation of these discrepancies, but for the present the resolution of this problem is beyond the scope of our work. Some of the difficulties might be resolved in a practical setting by stratifying the data, which so far we have not done.

The Jolly-Seber model makes strong assumptions, particularly concerning temporary emigration whence verification of results by a second method such as ours is desirable. Ideally we would like to combine the Jolly-Seber type estimators with the log-linear estimators. The 
development of a dynamic continuous time model extending the previous work of Farcomeni \& Scacciatelli (2013) so as to allow multiple capture types would be useful. However, the complex interactions between the observation methods within a year suggest this would be challenging. Section 5 of Cormack (1989) shows that the log-linear model can be applied to open populations, but a gain with multiple lists the modelling exercise becomes formidable.

There are many recent approaches to variable selection in generalised linear models that may be considered in practice to reduce the variance of the estimated population size (e.g., Calcagno \& Mazancourt 2010; Friedman et al. 2010; McLeod \& Xu 2014; You $\&$ Mao 2009). Moreover the resulting models themselves may be of interest. In the event of overdispersion we may consider the quasi-Poisson approach and because our remark at the end of Section 2 still holds in this context our approach remains valid. The negative binomial model is also applied commonly to overdispersed data, but in this case our remark does not apply. Consequently although we can estimate the number of unobserved drug users under the negative binomial approach we encounter the same difficulties as Liu et al. (2007) in estimating the standard error of the total numbers of drug users.

Finally, we note that our cross-sectional estimators arise from a composite marginal likelihood. This indicates how the approach may be extended to inference on the population dynamics. Composite likelihood is a well known technique used to simplify complex likelihoods. See Varin et al. (2011) for a recent review. Set in this framework, for parameters $\theta_{1}, \ldots, \theta_{J}$ we could maximise

$$
\mathcal{L}\left(\boldsymbol{\theta}_{1}, \ldots, \boldsymbol{\theta}_{J} ; \boldsymbol{y}_{J}, \ldots, \boldsymbol{y}_{1}\right)=\prod_{i=1}^{J} f\left(\boldsymbol{y}_{i} ; \boldsymbol{\theta}_{i}\right)
$$

with respect to $\boldsymbol{\theta}_{1}, \ldots, \boldsymbol{\theta}_{J}$ where $f\left(\boldsymbol{y}_{i} ; \boldsymbol{\theta}_{i}\right), i=1, \ldots, J$ are the marginal densities arising from the annual observations. Let $\widehat{\boldsymbol{\theta}}_{i}, i=1, \ldots, n$ denote the resulting estimators. We could use these to estimate the population size. Inference on the population dynamics could be conducted using the two-stage approach of Zhao \& Joe (2005). For example, let $f\left(\boldsymbol{y}_{i}, \boldsymbol{y}_{j} ; \boldsymbol{\theta}_{i}, \boldsymbol{\theta}_{j}, \gamma_{i j}\right)$ be the joint density of $\boldsymbol{y}_{i}$ and $\boldsymbol{y}_{j}$, where $\gamma_{i j}$ is the vector of additional parameters required to fully specify the joint density. Write $\boldsymbol{\theta}=\left(\boldsymbol{\theta}_{1}^{\top}, \ldots, \boldsymbol{\theta}_{n}^{\top}\right)^{\top}$ and $\gamma=\left(\gamma_{i j}^{\top}, i=1, \ldots, n-1, j=i+1, \ldots, n\right)^{\top}$. Then inference on $\gamma$ could be based on the pairwise composite likelihood evaluated at the $\widehat{\boldsymbol{\theta}}_{j}$. That is,

$$
\mathcal{L}_{2}\left(\widehat{\boldsymbol{\theta}}, \boldsymbol{\gamma} ; \boldsymbol{y}_{n}, \ldots, \boldsymbol{y}_{1}\right)=\prod_{i=1}^{n-1} \prod_{j=i+1}^{n} f\left(\boldsymbol{y}_{i}, \boldsymbol{y}_{j} ; \widehat{\boldsymbol{\theta}}_{i}, \widehat{\boldsymbol{\theta}}_{j}, \boldsymbol{\gamma}_{i j}\right)^{w_{i j}}
$$

for weights $w_{i j}, i=1, \ldots, n-1, j=i+1, \ldots, n$. One possibility for the weights that results in inferences being based on adjacent time points is $w_{i, i+1}=1$ and $w_{i, j}=0$ for $j \neq$ 
$i+1$. This extension requires the development of new models for the joint densities and will be the subject of future work.

\section{References}

Calcagno, V. \& de Mazancourt, C. (2010). glmulti: An R package for easy automated model selection with (generalized) linear models. J. Statist. Soft 34, 1-29.

Cormack, R.M. (1989). Log-linear models for capture-recapture Biometrics 45, 395-413.

Davison, A.C. \& Hinkley, D.V. (1997). Bootstrap Methods and Their Application. New York: Cambridge University Press.

European Monitoring Centre for Drugs and Drug Addiction (1997). Estimating the prevalence of problem drug use in Europe. Scientific Monograph Series, Number 1. Lisbon: European Monitoring Centre for Drugs and Drug Addiction.

Eilers, P.H.C. \& Marx, B.D. (1996). Flexible smoothing with B-splines and penalties. Statist. Sci. 11, 89-121.

Farcomeni, A. \& Scacciatelli, D. (2013). Heterogeneity and behavioral response in continuous time capturerecapture, with application to street cannabis use in Italy. Ann. Appl. Statist. 7, 2293-2314.

Fienberg, S. E. (1972). The multiple recapture census for closed populations and incomplete $2^{k}$ contingency tables. Biometrika 59, 591-603.

Friedman, J., Hastie, T. \& Tibshirani, R. (2010). Regularization paths for generalized linear models via coordinate descent. J. Statist. Soft 33, 1-22.

Hickman, M., Taylor, C., Chatterjee, A., Degenhardt,L., Frischer, M., Hay, G., Tilling, K., Wiessing, L., Griffiths, P. \& McKetin, R. (2002). Estimating the prevalence of problematic drug use: a review of methods and their application. Offprint from Bulletin on Narcotics, vol. LIV, Nos 1 and 2.

Huggins, R.M. \& Yip, S.F.P. (1999) Estimation of the size of an open population from capture-recapture data using weighted martingale methods. Biometrics 55, 387-395.

International Working Group for Disease Monitoring and Forecasting (1995a). Capture-recapture and multiple-record systems estimation I: History and theoretical development. Am. J. Epidemiol. 142, $1047-1058$.

International Working Group for Disease Monitoring and Forecasting. (1995b). Capture-recapture and multiple-record systems estimation II: Applications in human diseases. Am. J. Epidemiol. 142, 10591068.

Jolly G.M. (1965). Explicit estimates from capture-recapture data with both death and immigration-stochastic model. Biometrika 52, 225-247.

Liu, D., Yip, S.F.P. \& Huggins, R.M. (2007). Nonparametric method for estimating the size of an open population using marginal data from repeated multiple lists. Aust. N. Z. J. Statist. 49, 303-320.

Marra, G. \& Wood, S.N. (2012). Coverage properties of confidence intervals for generalized additive model components. Scand. J. Statist. 39, 53-74.

McLeod, A.I. \& Xu, C-J. (2014). bestglm: Best subset GLM. R-package version 0.34. http: / /CRAN . $\mathrm{R}$-project.org/package=bestglm.

Policy 21 Limited. (2013a). A review of estimation methods on prevalence of drug abuse population in Hong Kong. Final Report.

Policy 21 Limited. (2013b). A review of estimation methods on prevalence of drug abuse population in Hong Kong. Executive Summary.

Pollock, K.H. (1982). A capture-recapture design robust to unequal probability of capture. J. Wildl. Manage. 46, 752-757.

R Core Team (2016). R: A language and environment for statistical computing. R Foundation for Statistical Computing, Vienna, Austria. http://www.R-project.org/.

Seber, G.A.F. (1965). A note on the multiple-recapture census. Biometrika 52, 249-259. 
Varin, C., Reid, N. \& Firth, D. (2011) An overview of composite likelihood methods. Stat. Sinica 21, 5-42. Venables, W.N. and Ripley, B.D. (2013). Modern applied statistics with S-PLUS. New York: Springer-Verlag. Wood, S.N. (2006). Generalized Additive Models: An Introduction with R. Boca Raton, Florida: Chapman \& Hall/CRC.

You, N. \& Mao, C.X. (2009). On hierarchical loglinear models in capture-recapture studies. Comp. Statist. Data Anal. 53, 3916-3920.

Zhao, Y. \& Joe, H. (2005) Composite likelihood estimation in multivariate data analysis. Canad. J. Statist. 33, 335-356

Zwane, E.N., Van Der Pal-De Bruin, K. \& Van Der Heijden, P.G.M. (2004). The multiple-record systems estimator when registrations refer to different but overlapping populations. Stat. Med. 23, 2267-2281.

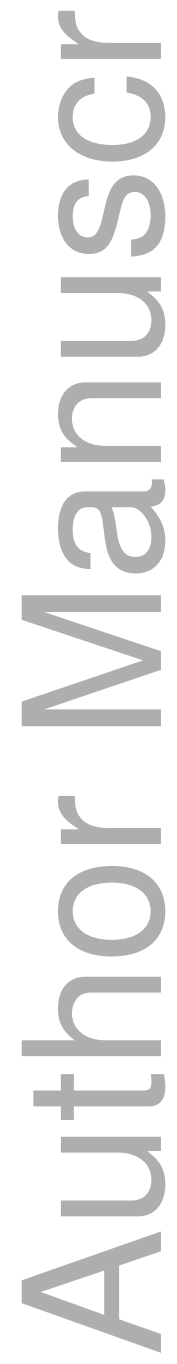




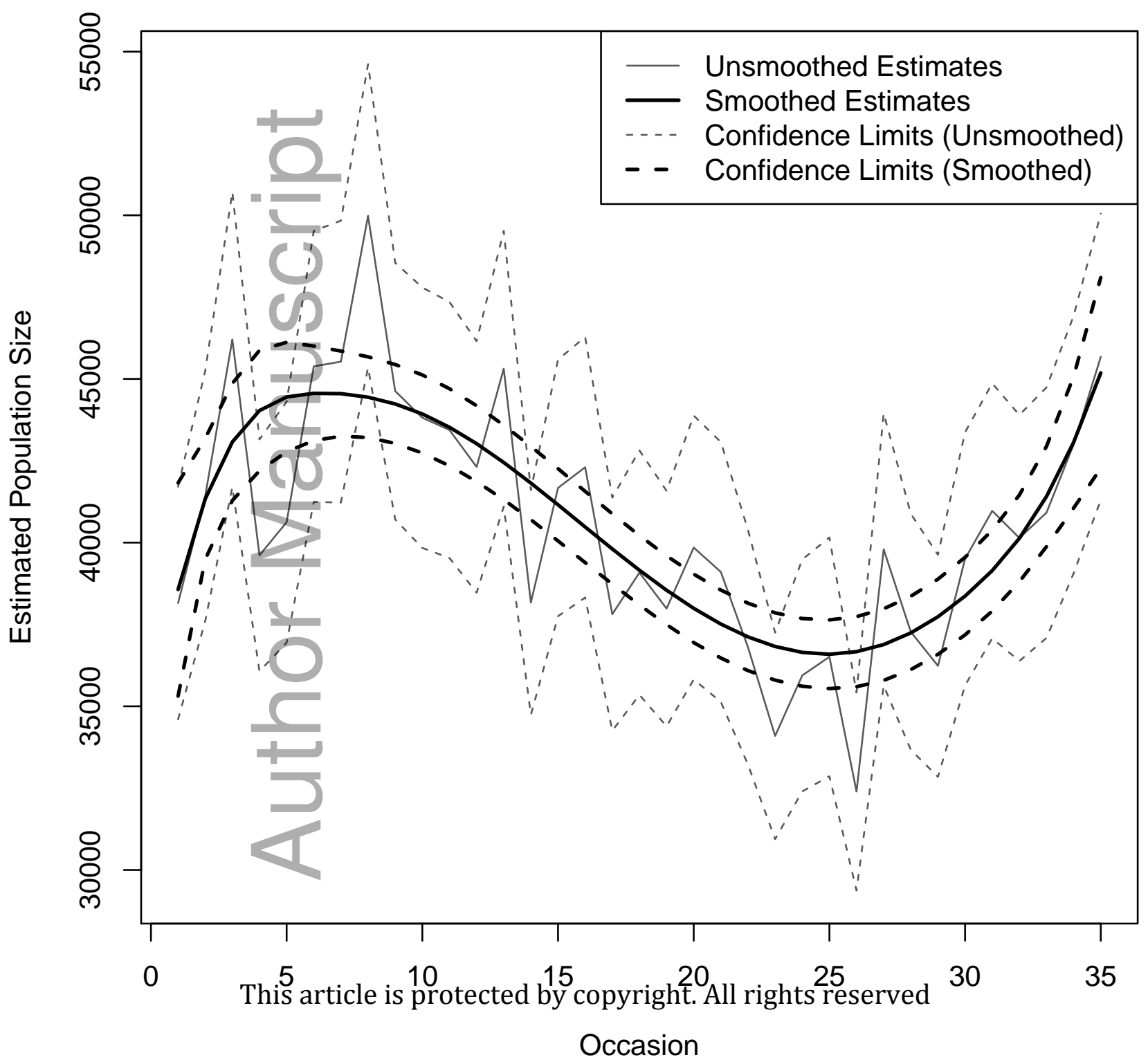


anzs_12149_f2.pdf

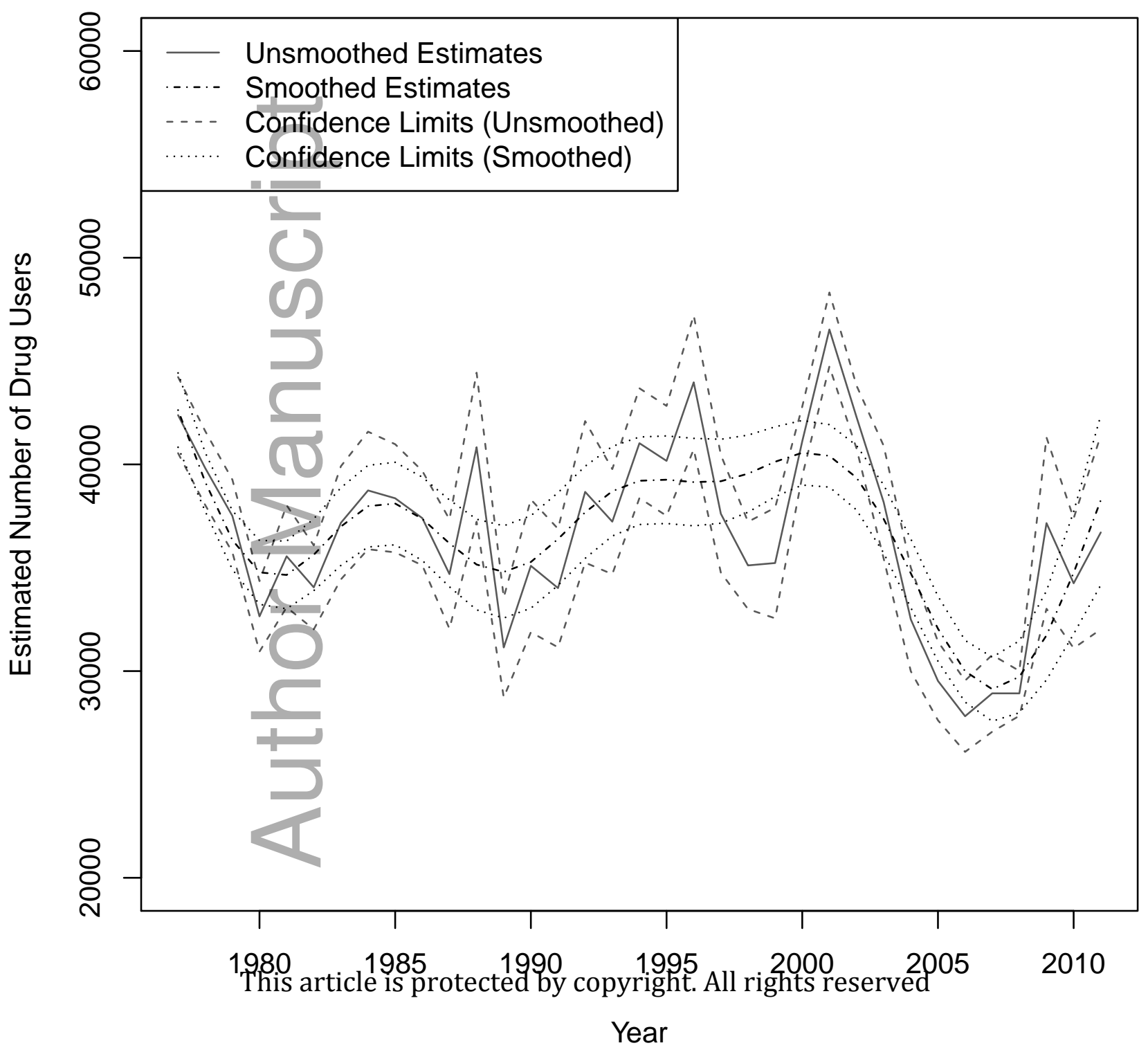




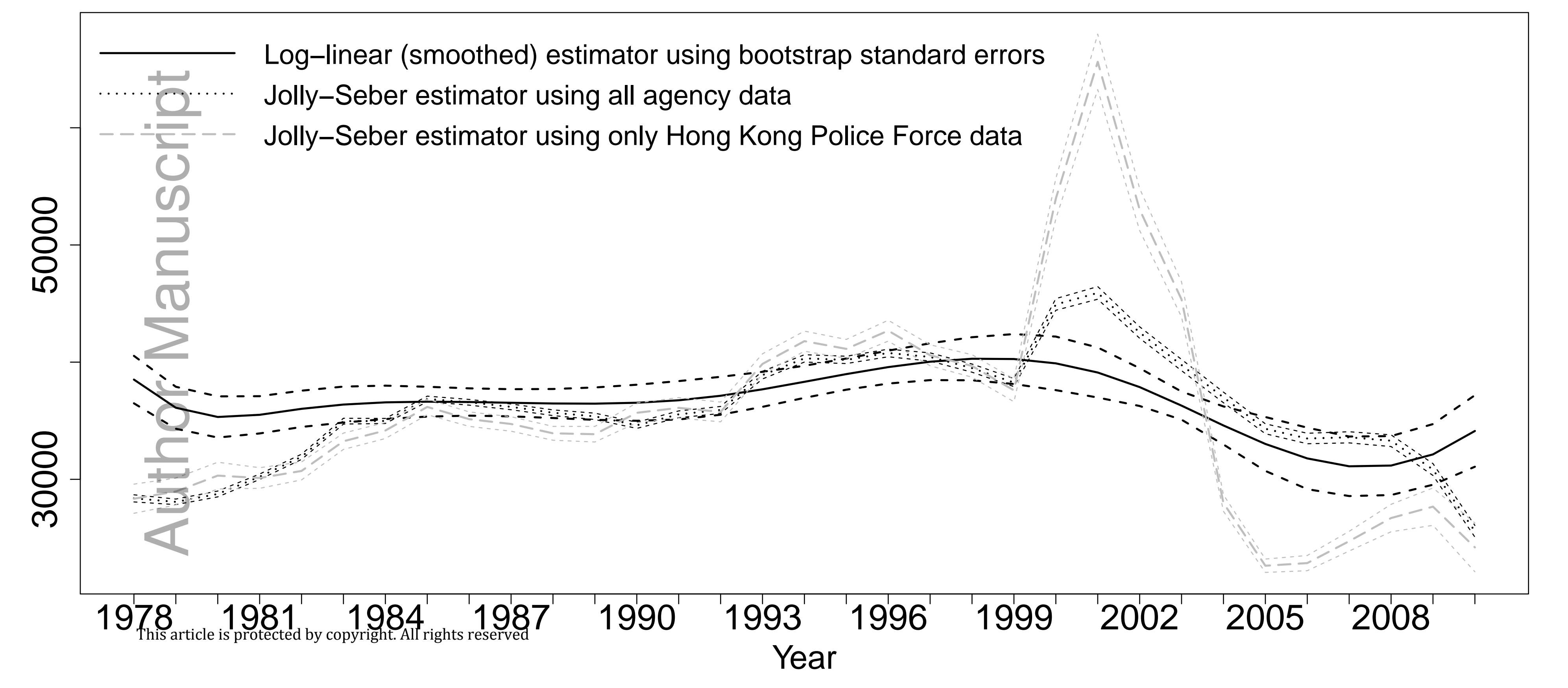




\section{University Library}

\section{- M M N E R VA A gateway to Melbourne's research publications}

Minerva Access is the Institutional Repository of The University of Melbourne

Author/s:

Huggins, RM;Yip, PSF;Stoklosa, J

Title:

Nonparametric Estimation of the Number of Drug Users in Hong Kong Using Repeated Multiple Lists

Date:

2016-03-01

Citation:

Huggins, R. M., Yip, P. S. F. \& Stoklosa, J. (2016). Nonparametric Estimation of the Number of Drug Users in Hong Kong Using Repeated Multiple Lists. AUSTRALIAN \& NEW ZEALAND JOURNAL OF STATISTICS, 58 (1), pp.1-13. https://doi.org/10.1111/anzs.12149.

Persistent Link:

http://hdl.handle.net/11343/291011 